\title{
Comparison of Multiple Stage Braking Circuits for Wind Driven Generators
}

\author{
Plamen Valentinov Yankov, Vencislav Cekov Valchev \\ Department of Electronic Engineering and Microelectronics \\ Technical University of Varna \\ Varna, Bulgaria \\ plamenvalentinov@tu-varna.bg
}

\begin{abstract}
This paper presents multiple stage braking circuits for wind turbines with a permanent magnet synchronous generator. The system combines both passive converter circuit coupled to a resistive braking circuit. The second one is an over voltage protection when an emergency situation of no load is detected. The power converter control and braking are simulated and the output power/frequency graphics are derived. Three braking circuits are analyzed and compared. Design recommendations are formulated regarding the application of different topologies depending of the wind speed and the input assignments.
\end{abstract}

Keywords-braking system, passive converter, simulation, wind driven generator

\section{INTRODUCTION}

Depending on the size of the wind turbine there are several solutions to reduce its speed or stop it at stand still. The mechanical brake is essential but once used in emergency situation it might need a proper maintenance in order to be reused. Another common method is by using aerodynamic properties of the blades (pitch control, active and passive stall control, etc.) Those systems add weight at the nacelle but they can be used multiple times and can improve the energy yield. The third option to brake a wind turbine is to use power electronics to control the electrical loading on the generator. This can be achieved by applying different schematics and control strategies. One of the possible solutions is to use the kinetic energy of the rotor in the event of the utility grid failure to charge a supercapacitor. Then the stored energy is used to power the braking circuit and stop the wind turbine in electrodynamic way [1]. Usually this circuit is realized with a transistor and pulse width modulation control and is often called "chopper" brake circuit positioned on the DC-link [2]. However, the use of transistors has some disadvantages such as high levels of electromagnetic interference (EMI), and also the use of complicated control unit can lead to an undesired reliability issues.

This paper suggests the use of passive braking circuit in order to reduce or completely remove previously mentioned downsides [3]. The thyristor used as power electronic switch in the passive braking circuit start to conduct in the event of utility grid failure. It stays switched "on" through the whole process of braking, so there isn't any high frequency

\author{
Alex Van den Bossche \\ Department of Electrical Energy, Systems and Automation \\ Ghent University \\ Ghent, Belgium \\ alex.vandenbossche@ugent.be
}

commutation and therefore significantly reduced EMI rates. The triggering circuit includes only passive components and no microcontroller, thus a simple analog circuit results in a robust and reliable design.

The overall structure is depicted on figure 1. The blocks on the figure are as follows:

PMSG - permanent magnet synchronous generator. As most advanced type of generator up to the moment, it is used as way of reaching even higher efficiency and power output of wind turbines [4,5]. Because the excitation is done by permanent magnets all the generated electricity is converted by power electronics rated for the whole output power of the designed wind turbine.

PEC1 - the first power electronic converter is a passive converter circuit (rectifier). Using two full wave three phase rectifiers and externally connected inductors this circuit has some significant advantages over the conventional back to back converters. The circuit uses diodes instead of transistor to form a power/frequency output curve that is as close as possible to the ideal cubic dependence of the wind speed and power output of the machine [6].

Lext - external inductors connected to the first power electronic converter. Their values are determined by methodology which ensures the highest energy yield from the wind turbine generator. An automation of the methodology gives fast design approach of the reactive components in the passive converter circuit [7].

EB - electric brake block represents the passive braking circuits which are under test in paper. It is positioned at the DC-link level. It is the most efficient way of stopping the wind turbine during electrical grid failure and prevents physical damage of the structure due to uncontrolled spin of the hub, blades and rotor.

PEC2 - second power electronic converter, which is an inverter circuit. This block is not discussed in the paper because of the nature of the simulation, precisely utility grid failure.

The main blocks which concern this research are marked with red dashed line. As most spread and efficient design on figure 1 is depicted three bladed wind turbine. 


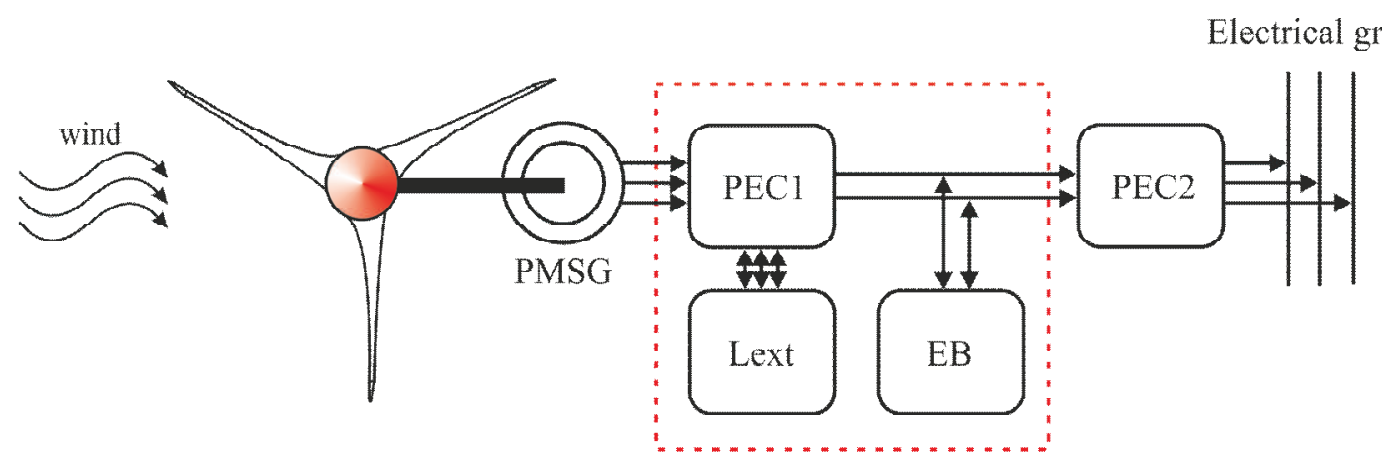

Fig. 1. Structure of the device under test.

\section{BASIS OF THE PASSIVE BRAKING CIRCUIT}

On figure 2 is depicted the overall schematic of the single stage passive braking circuit connected to a passive converter circuit. The electric generator is represented as three voltage sources (AC 1, AC 2 and AC 3) and three inductors (L1, L2 and L3). The methodology for design of the passive converter circuit includes proposition for the generator winding values. All the windings are externally connected through external inductors (L4, L5 and L6). The rectifier consists of a two three phase uncontrolled rectifiers connected to every winding at both ends of the three phase generator. Simulations shows that external inductors improve significantly low speed power output of the wind turbine [6].

After the DC link capacitor (represented with a capacitor and resistor for its ESR parameter) the single stage braking circuit is positioned. The main power components are the power electronic switch - thyristor Th1 and a dissipating resistor Rdiss, which spreads the energy from the braking process into the surrounding environment. The triggering circuit acts automatically and consists of two resistors R1 and $\mathrm{R} 2$, a zener diode DZ1, capacitor C2 and a diac DK1.

When a grid failure occurs the DC link voltage rises and the triggering circuit detects that with DZ1. The zener diode braking voltage is chosen to be the nominal operation voltage. When the voltage rises above nominal DZ1 starts to conduct and therefore charges the capacitor $\mathrm{C} 2$ trough resistor $\mathrm{R} 1$. The diac DK1 is triggered "ON" when the voltage across C2 reaches $32 \div 50$ volts (depending on the diac trigger voltage). Resistor R2 limits the current which flows through the gate of the thyristor Th1. After this the thyristor starts to conduct and all of the electrical energy from the generator is dissipated as heat by the resistor Rdiss.

The negative torque applied by the electrical machine needs to be higher than nominal operation torque at every wind speed in order to stop the rotating shaft. For example a $100 \mathrm{~kW}$ wind turbine with DC link at 1000 volts will have current of 100 amperes. So the resulting impedance will be:

$$
\text { Znom }=\frac{U}{I}=\frac{1000}{100}=10 \Omega
$$

To ensure the braking torque needed the value of the dissipating resistor is between half of Znom and Znom.

The circuit has the advantage of simplicity, robustness and low EMI values compared to a conventional "chopper" brake. However at medium and high rated power of a wind turbine, braking with single stage can lead to a significant instant negative torque load on the shaft and the gearbox. A solution to this problem is proposed by introducing multiple stage braking with several preset dissipation resistors. Figure 3 presents a triple stage passive braking circuit.

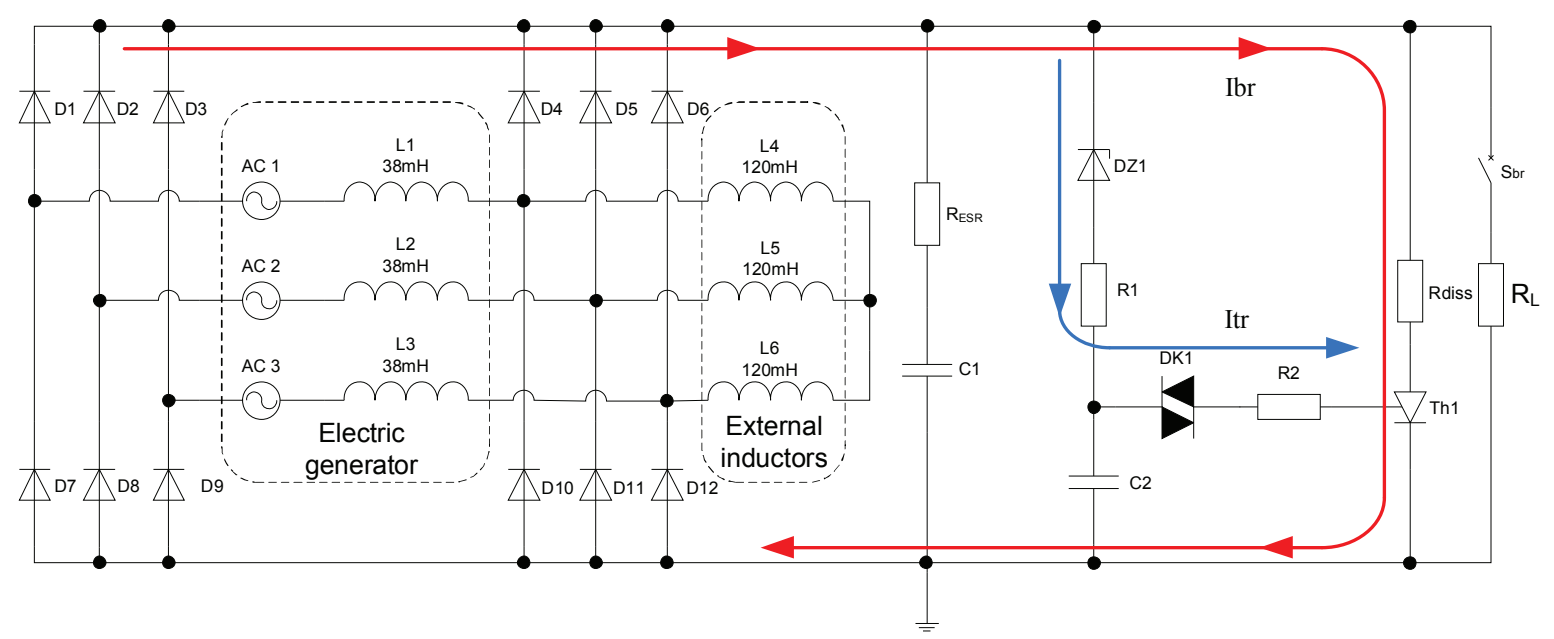

Fig. 2. Single stage passive braking coupled to a passive converter circuit. 


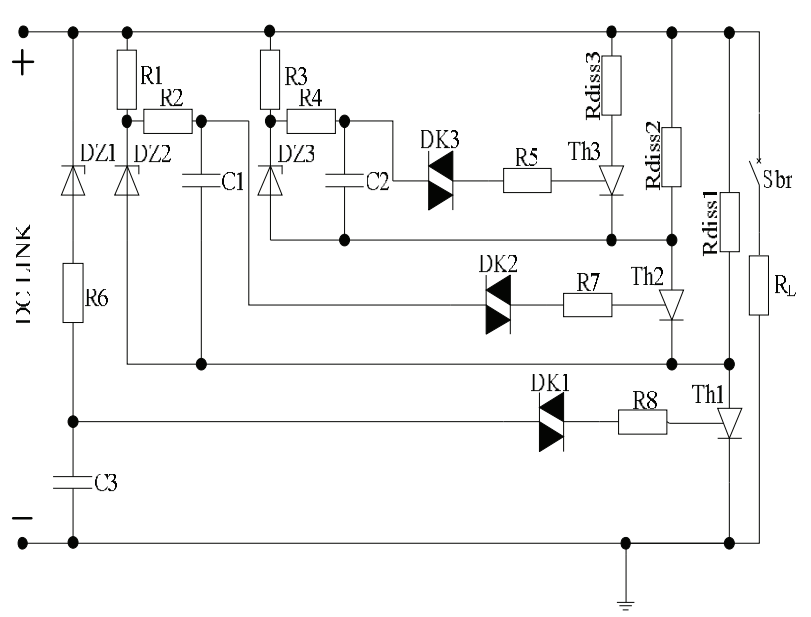

Fig. 3. Three stage passive braking circuit.

\section{Multistage Passive BRAKING CIRCUIT}

The first stage of the multiple braking circuit operates as already explained in the paper. However the second, third and etc. stages, trigger their thyristors when two conditions are fulfilled. Firstly, when thyristor Th1 is switched "ON" by a grid failure, it provides path for the current which passes through the next stage thyristor - in this case thyristor Th2. The triggering circuit on the next stages differs from the first one. It uses predefined timeout, set by a RC time constant circuit. For second stage it is determined by $\mathrm{R} 2$ and $\mathrm{C} 1$ and for third stage by $\mathrm{R} 4$ and $\mathrm{C} 2$.

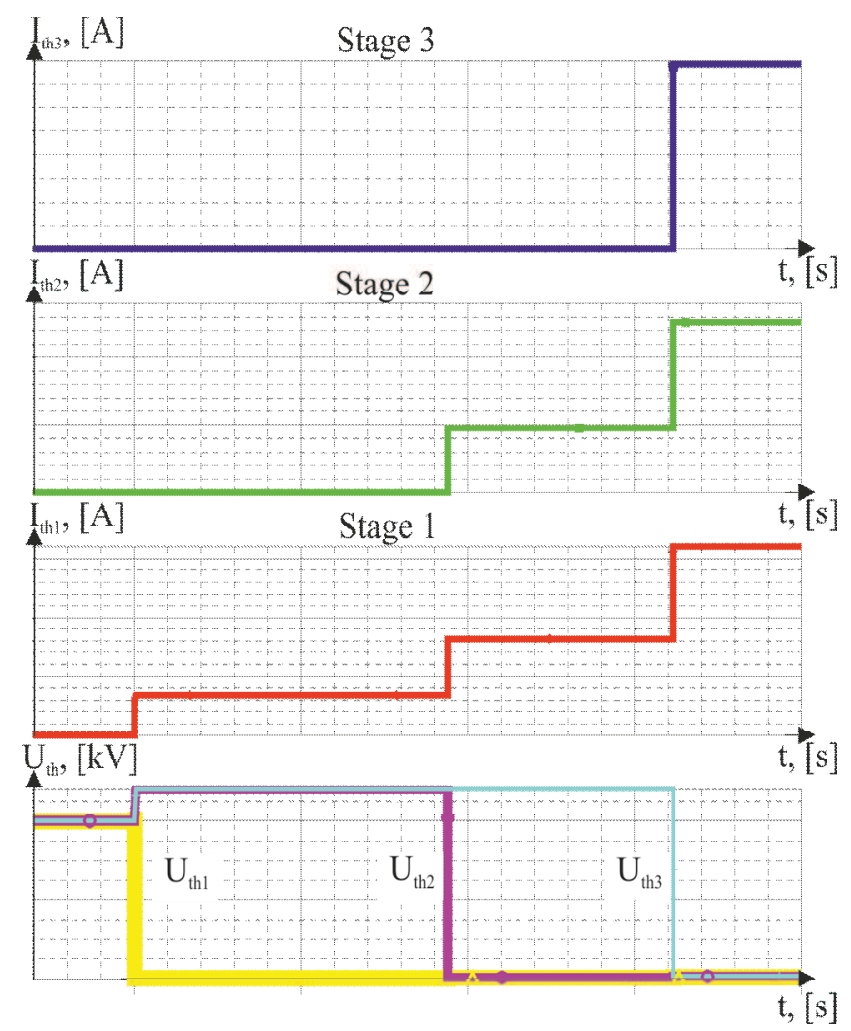

Fig. 4. Triggering process of multiple stage passive braking circuit.
The resistors R1 and R3 limit the current through the zener diodes ZD2, ZD3 (their braking voltage is chosen to be 51 volts) and the rest of the triggering circuit. When capacitor $\mathrm{C} 1$ is charged to the breakover voltage of diac DK2, thyristor Th2 is turned on. This same sequence works for every other stage of the braking circuit.

Figure 4 presents time diagram of the switching sequence of all thyristors. A sample of currents and voltages across the thyristors through braking process are given. Dissipation resistors are with three different values. As previously mentioned example, $100 \mathrm{~kW}$ wind turbine has dissipation resistors in the range between $5 \div 10 \mathrm{ohms}$. The simulations are done with the following values of the dissipation resistors on the corresponding stages Stage 1-Rdiss $1=5 \Omega$, Stage 2 - Rdiss2 $=4 \Omega$ and Stage $3-$ Rdiss $3=3 \Omega$.

The outcome of this figure gives impression of rising current trough the thyristors except on the last stage. In fact when reducing the speed of the generator the output power is lower, so the current and voltages are lower. The simulations in OrCAD are done without feedback, so the generator continues to rotate and produce energy even at Stage 3 of the braking process. A future work on MATLAB Simulink model can improve this and give the interaction between electrical circuits and mechanics.

The actual braking process of a single stage braking circuit can be observed on figure 5. Three different dissipation resistors are used to form braking curves - Pbr1, Pbr2, and $\mathrm{Pbr} 3$. The graphic compares those power/frequency curves with the nominal operation mode curve of the passive

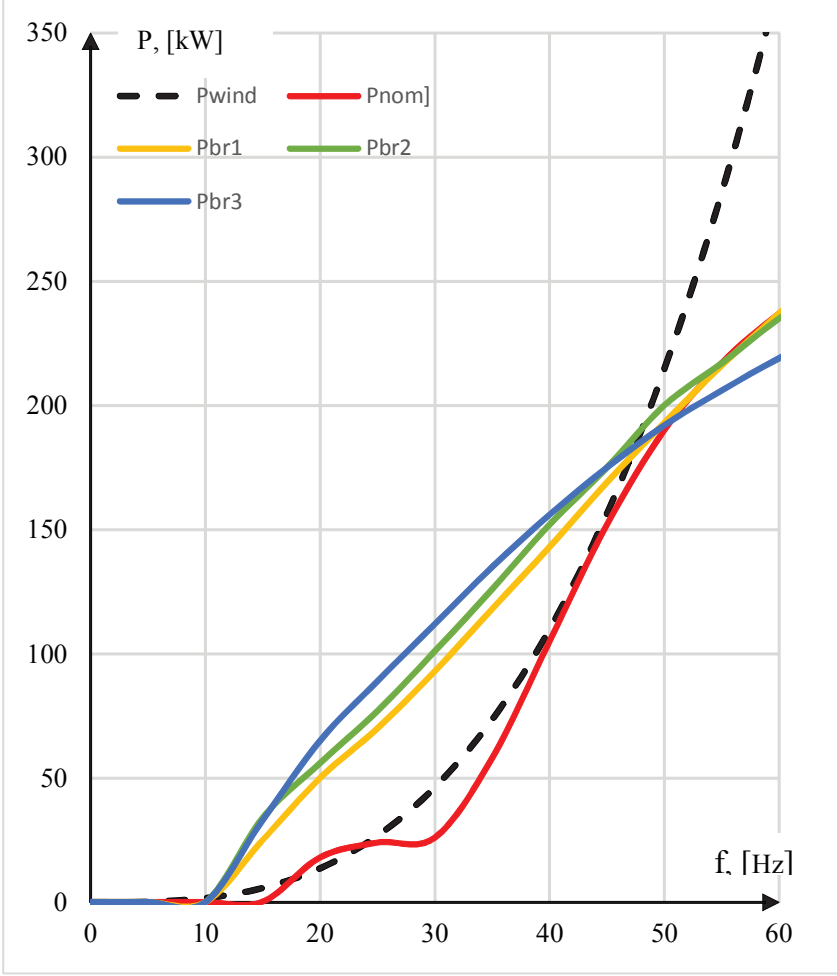

Fig. 5. Braking curves of single stage passive braking circuit. 
converter circuit - simulation of $100 \mathrm{~kW}$ variable speed wind turbine. Last curve marked with dashed line shows the cubic dependence between power and wind speed and can be expressed with the following equation:

$$
P=\frac{1}{2}(\rho A V) V^{2}
$$

Figure 6 shows the output braking curve of the three stage braking circuit. The values of the dissipating resistors are the same as in the single stage simulation. At nominal operation range of the simulated variable speed wind turbine (from $15 \div 40$ hertz) it can be observed that there is significant braking power (torque). More specifically at the range of $35 \div 40 \mathrm{~Hz}$ there are correspondingly from $100 \%$ to about $35 \%$ more electrodynamical load than the power from the kinetic energy

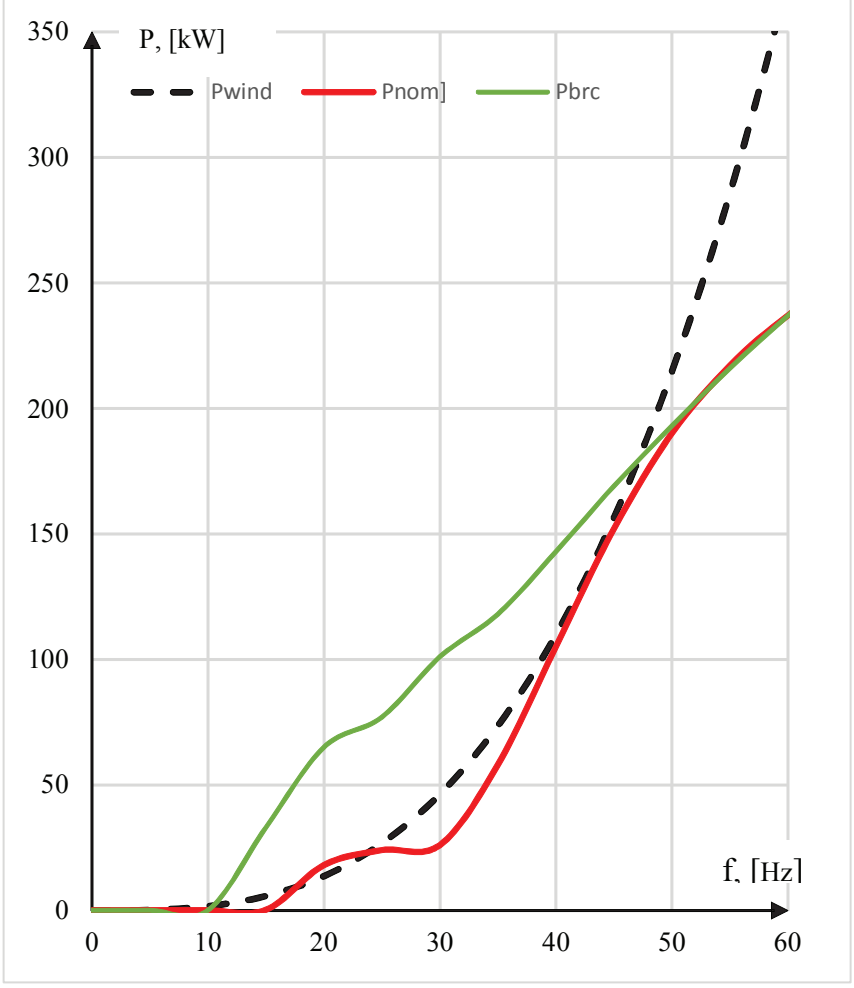

Fig. 6. Braking curve of three stage passive braking circuit. from the wind. At lower speed of the rotor there is more than $200 \%$ loading above nominal operation mode. This ensures fast and secure braking process at low wind speed.

As it can be seen from figures 5 and 6 , the passive braking circuit can be applied with successes only at nominal and below nominal wind speed of the wind turbine in the study. The design is applicable at medium and large sized wind turbine generators.

\section{CONCLUSION}

The outcome of the carried out simulations shows a definitive wind speed/frequency range in which the passive braking circuit is capable of reducing the rotational speed of a wind turbine during utility grid failure. The research recommends the use of a multiple stage braking circuit for medium and high power ratings. This will reduce the negative torque stress on shafts and gearbox. Also the electric brake gives the advantage of multiple usage thus less maintenance cost.

\section{REFERENCES}

[1] J. Lyons, A. Esser, P. Bixel, "Method and system for wind turbine braking”, US Patent 5907192 A, May 25, 1999.

[2] T.C.Y. Wang, W. Yang, X. Yuan, R. Teichmann, "A redundant electrical braking system for wind turbine generators", EPE 2007, Article number 4417293

[3] P. Yankov, A. Van den Bossche, V. Valchev, "Successive resistive braking circuit for permanent magnet wind turbine generators", EPEPEMC'2010, 6-8 September, Ohrid, Macedonia, 2010, ISBN 978-14244-7854-5, pp. T11-27 $\div$ T11-31;

[4] K. Ahsanullah, R. Dutta, M.F. Rahman, "Review of PM generator designs for direct-drive wind turbines", AUPEC 20122012, Article number 6360197.

[5] A. Van den Bossche, P. Yankov, V. Valchev, "Design of Passive Converter for Wind Driven Generators", EPE-PEMC'11, Birmingham, UK, 30 August - 1 September, 2011, ISBN/ISSN: 978-1-61284-167-0, pp. 1-10.

[6] A. Van den Bossche, P. Yankov, A. Marinov, "Automated methodology for adjustment of component values in passive converter circuit for wind turbine generators", EPE-PEMC, 4-6 September, 2012, Novi Sad, Serbia, ISBN: 978-1-4673-1971-3, DS2d.4-1 -DS2d.4-4.

[7] O. Carranza, E. Figueres, G. Garcerá, “Analysis of the control structure of wind energy generation systems based on a permanent magnet synchronous generator", Applied Energy Volume 103, March 2013, Pages 522-538. 